\title{
Diagnostic Procedures and Follow Up of Macular Microstructural Changes in Patients with Uveitis as Seen on Optical Coherence Tomography
}

Jelena Paovic ${ }^{1^{*}}$, Predrag Paovic ${ }^{1}$, Anka Stanojevic Paovic ${ }^{2}$ and Vojislav Sredovic ${ }^{3}$

${ }^{1}$ Department of Ophthalmology, University Clinical Center, Pasterova 2, 11000 Belgrade, Serbia

${ }^{2}$ Uvea Center, Center for Diagnosis and Treatment of Eye Diseases, Kneza od Semberije 14, 11000 Belgrade, Serbia

${ }^{3}$ Primary Health Care Center, Jove Negusevica 5, 22419 Pecinci, Serbia

\begin{abstract}
The aim of this study was to show how optical coherence tomography (OCT) can provide means for assessing macula and the optic disc, and so can be used to diagnose and follow micro-structural, macular, changes (epiretinal membranes; macular edema; lamellar hole and discontinuity of inner/outer segment line), that occur as complications of a disease (i.e. uveitis). It is a non invasive, non contact method that provides cross-sectional images of retina and its substructures in a real-time mode. High definition cross-sectional images obtained by a commercially available OCT are attained at 1-15 $\mu \mathrm{m}$ and can provide detailed pictures from deep within the retina. Seeing that OCT can be repeated numerous times it is useful for practitioners that need to follow treatment effects and progression of the disease.
\end{abstract}

Spectral-Domain OCT was used to diagnose and follow macular changes in patients with uveitis (intermediate and posterior) and retinal vasculitis. OCT showed an epiretinal membrane (ERM) as intense back-reflective layer on the inner retinal surface. Via this method thickness measurements of ERM as well as the degree of inner retinal surface attachment (focal or diffused) were acquired and precisely defined. OCT showed macular edema as retinal layering followed by presence of intraretinal cavities and absence of back-reflection. It provided means of measuring thickness, localization, as well as size of intraretinal cysts during the evolution of macular edema (ME), and so was utilized in analysis of various factors such as progression of ERM; presence of ME; surface roughness; changes of thickness and layer configuration of retinal tissue.

OCT macular scan provides precise and quick diagnosis of delicate microstructural retinal changes in high definition that can't be obtained during classical clinical examination. Progression of disease, changes and treatment effects, followed via OCT, are of great importance in clinical practice.

Keywords: Microstructural macular changes; Epiretinal membrane; Macular edema; Lamellar hole; Optical coherence tomography; Uveitis; Follow up

\section{Introduction}

Uveitis is an immune or autoimmune disorder of uveal tissue, of infectious/non-infectious genesis, most commonly bilateral and recurrent. If left untreated it can lead to significant decrease in visual acuity or even to permanent visual loss. According to localization uveitis can be classified as: anterior, intermediate, posterior and total. Macular disorders are the most severe complications of intermediate and posterior uveitis and have substantial consequences on visual acuity [1-5]. Clinical diagnosis of macular disorders is based on biomicroscopy and indirect ophthalmoscopy; fluorescein angiography (FA); indocyanine-green angiography (ICG angiography); optical coherence tomography (OCT) and/ biopsy of uveal and retinal tissue [6,7]. Pathological processes occurring in the macula can be assumed via direct and indirect ophthalmoscopy based on aspect of macular surface and its reflexes such as: epiretinal membranes (ERM); lamellar hole; macular edema (ME); subretinal macular edema (SME); choroidal neovascularization (CNV) etc. Invasive procedures such as FA and ICG are used in assessment of hematoretinal barrier disruption and CNV evolution [8]. These methods provide more precise data on evolution and presence of CNV as compared to OCT but they on the other hand are not as good in diagnosis and follow-up of intraretinal and subretinal edema and evolution of EM. Practically, we have a situation where OCT provides more detailed information of the deep tissue as well as histological changes that might have occurred. In some cases FA and ICG will make note of histological changes in the affected macular tissue but they will not be able to provide data on the extent of these structural changes. OCT, as medical imaging system, is a non invasive, non contact, technique which provides crosssectional images of the retina and its substructures in a real-time mode. High definition cross-sectional images obtained by OCT are attained at 1-15 $\mu \mathrm{m}$ and provide superior detail when compared to standard B-scan ultrasonography, magnetic resonance imaging (MRI) and/ computerized axial tomography (CAT scan). Macular and optic disc structures, in situ, and/ treatment efficiency, can be seen, followed, and evaluated through repeated use of this method [9-11]. One of the first OCT images of human retina, in vivo, was taken in the early 90's. [12].

OCT devices use continuous low-coherence light rays and are based on low-coherence interpherometry technique. In view of different optical characteristics of various scattering media, a ray of light is back-reflected and backscattered at different angles. Resolution of high definition OCT is $3 \mu \mathrm{m}$. Images obtained in this way can

*Corresponding author: Jelena Paovic, Department of Ophthalmology, University Clinical Center, Pasterova 2, 11000 Belgrade, Serbia, Tel: +38163 245552; E-mail: maliceda@eunet.rs

Received October 11, 2013; Accepted December 30, 2013; Published January 01, 2014

Citation: Paovic J, Paovic P, Paovic AS, Sredovic V (2013) Diagnostic Procedures and Follow Up of Macular Microstructural Changes in Patients with Uveitis as Seen on Optical Coherence Tomography. J Cytol Histol 5: 211. doi:10.4172/21577099.1000211

Copyright: ( 2014 Paovic J, et al. This is an open-access article distributed under the terms of the Creative Commons Attribution License, which permits unrestricted use, distribution, and reproduction in any medium, provided the original author and source are credited. 
Citation: Paovic J, Paovic P, Paovic AS, Sredovic V (2013) Diagnostic Procedures and Follow Up of Macular Microstructural Changes in Patients with Uveitis as Seen on Optical Coherence Tomography. J Cytol Histol 5: 211. doi:10.4172/2157-7099.1000211

be presented via grey or color logarithmic mapping thus showing intensity of back-reflected and backscattered signal. Axial OCT image resolution depends on a range of wave lengths, whilst transverse OCT image resolution is defined through the size of focused OCT ray [9].

Macular OCT images are interpreted via comparative study of data obtained both through OCT and through various histological findings (Figures 1a and $1 \mathrm{~b}$ ).

On a color-coded scale both neurofiber (NFL) and reticular layers (inner and outer) intensively back-reflect light and are shown in red. As opposed to this, granular layers back-reflect light poorly and are represented in blue-black. NFL is the first highly-reflective layer which can be seen along the inner retinal surface. This layer is thinner in the macular than in the optic disc region (where it has full retinal thickness). Three low intensity reflective layers are: retinal ganglion cell (RGC); inner and outer granular layers. RGC occurs around parafoveolar region. Mildly reflective are inner and outer reticular layers. Diagonal photoreceptor axon, which can sometimes be seen as an independent layer within the outer reticular layer (Henle layer), reflects high intensity light. Border at the junction of inner and outer photoreceptor segments is shown as a highly reflective thin line located above the retinal pigment epithelium (RPE) and choroidea. Reflective ability of this structure is probably due to a difference in reflection of various inner photoreceptor segments, and the extremely organized disc structures of the outer photoreceptors [10].

In the foveal region, as the outer layer photoreceptor cones increase in length, so does the thickness of the inner and outer photoreceptor segments. External limiting membrane, which can sometimes be seen as thin back-reflective layer and it is not an actual, physical, membrane, but rather a connective structure between photoreceptors and Müller cells. Melanin rich RPE is highly reflective. Bruch's membrane, located below RPE, ranging 1-4 $\mu \mathrm{m}$ in thickness, cannot be seen as separate structure. As it is closed to RPE, choriocapillar layer can't be seen clearly, eventhough it is highly reflective. Intense back-reflection of RPE leads to decreased visibility of deeper structures such as choroid and the choriocapillary layer (due to its shadow effect).

In some cases, retinal blood vessels can be visualized based on their intense reflection and on shadows caused by deeper structures. Eventhough they are seen as two separate highly reflective layers, border line between inner/outer photoreceptor segments and RPE/ choriocapillar complex on some OCT images can't be distinguished [9].
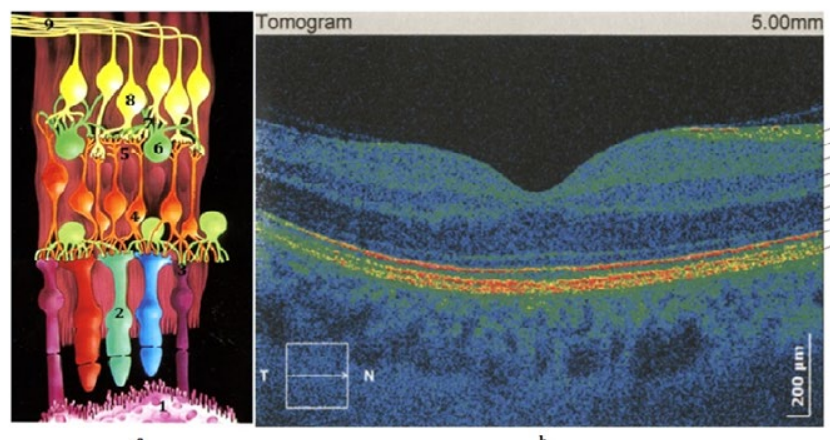

Figure 1: Human retina (a) scheme of retinal morphology, (b) OCT scan, macular region (HD-SOCT Copernicus + ), 1. Retinal pigment epithelium, 2. Inner and outer photoreceptor segments, 3. External limiting membrane, 4 Outer nuclear layer, 5. Outer plexiform layer, 6. Inner nuclear layer, 7. Inner plexiform layer, 8. Ganglion cell layer, 9. Neurofiber layer.
As OCT detects anterior (internal limiting membrane) and posterior layers (RPE), retinal thickness can be measured anywhere along the macula. Besides this, using data on thickness of various retinal points one can obtain its average, so that size, distribution and volume of retinal cysts can be measured and followed [11].

\section{Aim}

The aim of this study was to show how optical coherence tomography (OCT) can provide means for assessing macula and so can be used to diagnose and follow micro-structural macular changes (epiretinal membranes; macular edema; lamellar hole and discontinuity of inner/outer segment line), that occur as complications of a disease (i.e. uveitis)

\section{Methods}

Spectral-Domain OCT was used to diagnose and follow macular changes in patients with uveitis and retinal vasculitis. OCT provided data required for qualitative and quantitative analysis of microstructural changes of the macular tissue in diffuse and cystoid macular edema, subretinal liquid, and epiretinal membranes.

\section{Results and Discussion}

Use of OCT provides qualitative and quantitative data regarding macular structural changes, such as epiretinal membranes, intraretinal edema and lamellar hole, that are responsible for decreased visual acuity.

In patients with uveitis, epiretinal membranes have certain influence on visual acuity and may lead to development of monocular diplopia [13-15]. One of early signs of retinal deformation such as normal foveal contour loss can be attributed to ERM development, which can intern lead to formation of ME.

Epiretinal membrane is subsequent to posterior vitreal detachment, in cases where there is residual in the form of posterior vitreal capsule. In such cases, this leads to internal limiting membrane detachment and hyalocyte proliferation on its inner surface. This causes inner retinal surface contractions which are themselves follow by contraction of internal limiting membrane. Small break on internal limiting membrane occurs during posterior vitreal detachment and allows glial cells to proliferate along inner retinal surface thus forming ERM [1618]. Although ERM can be seen using direct/indirect ophthalmoscopy, OCT examination ensures higher level of sensitivity and thus better detection. Spectral-Domain OCT (SD-OCT) provides better visualization and higher insight of ERM and retinal changes which occur bellow it [19]. By using this method one can measure ERM thickness and precisely define degree of inner retinal surface attachment (focal or diffused). OCT shows ERM as intense back reflective layer on inner retinal surface. In cases where they are detached from retina, according to contour and intensity of reflection, ERM should be differentiated from detached hyaloid membrane. Thickness of ERM as well as its retinal tissue adhesion can vary during evolution of disease and course of treatment [20]. Figure 2a shows OCT scan of ERM with a thickness of $14 \mu \mathrm{m}$; distance from retina of $45 \mu \mathrm{m}$ temporally from foveal center and bridges fovea and forms retinal traction. Foveal layers are centrally displaced, while retina is layered without fluid accumulation. Figure $2 \mathrm{~b}$ shows retinal thickness displacement (black line) as compared to normal (green layer), where as Figure $2 c$ shows numeric and colorcoded average values of macular thickness correlated with an age of patient (blue-green represents normal finding, red-yellow represents pathological finding). ERM is focally attached to retinal surface in 
Citation: Paovic J, Paovic P, Paovic AS, Sredovic V (2013) Diagnostic Procedures and Follow Up of Macular Microstructural Changes in Patients with Uveitis as Seen on Optical Coherence Tomography. J Cytol Histol 5: 211. doi:10.4172/2157-7099.1000211

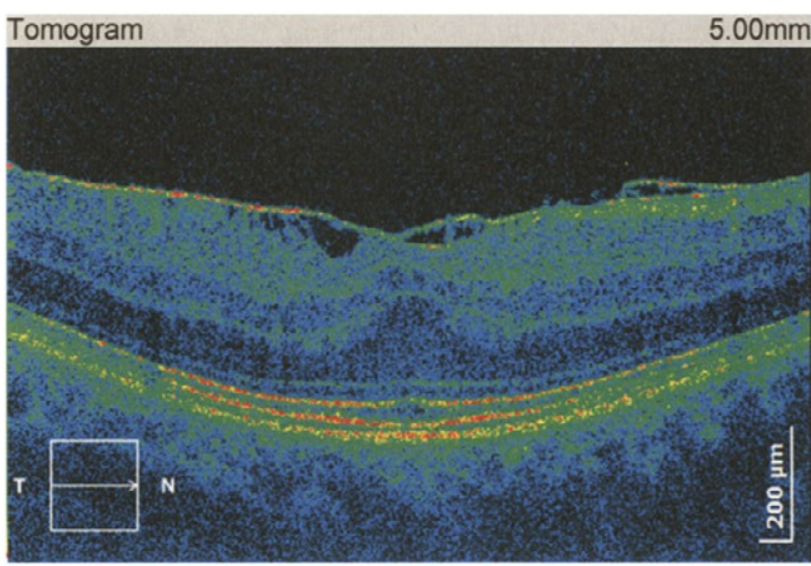

Figure 2a: OCT scan, epiretinal membrane in patient with uveitis.

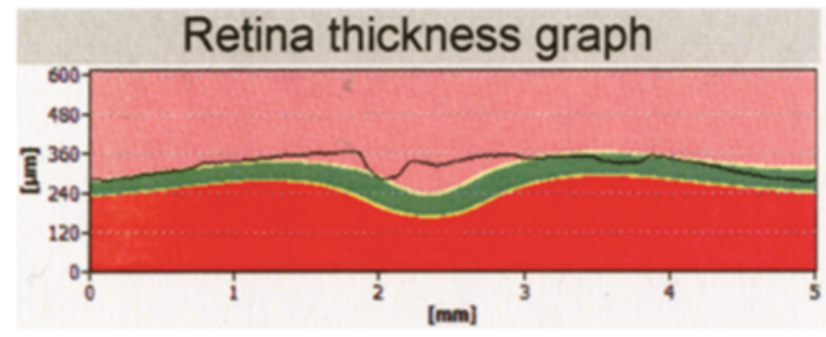

Figure 2b: Retinal thickness graph, epiretinal membrane in patient with uveitis.

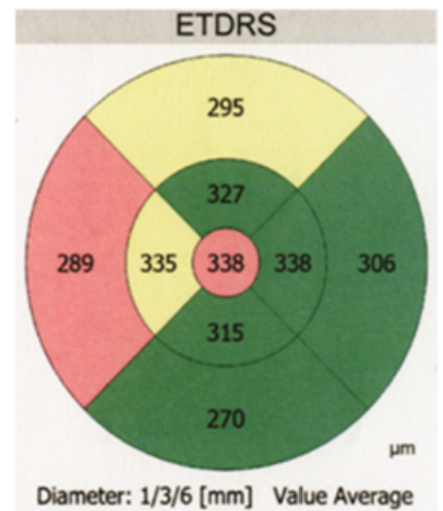

Figure 2c: Retinal thickness map - average value (diameter $1 / 3 / 6 \mathrm{~mm}$ ), epiretinal membrane in patient with uveitis.

the nasal region and sites of attachment are clearly defined (Figure 3). Due to ERM traction, retina is decomposed, thus allowing for fluid accumulation in the form of small cysts that can be seen due to absence of reflectance. Figure 4 shows OCT scan of pronounced ERM and diffused ME, distinct foveal contour elevation, retinal thickening as well as altered layers caused by severe ERM traction, with a central thickness of $31 \mu \mathrm{m}$ and temporal $109 \mu \mathrm{m}$. ERM is centrally attached to retina, which intern has diffused fluid accumulation occurring in its outer regions.

Macular edema occurs when fluid and protein deposits collect on or under the macula and cause it to thicken and swell. Macula is a retinal region rich in highly metabolic cells, and is a primary site for inflammatory, vascular and metabolic processes. Considering its anatomy macula is a predominant region for fluid accumulation where horizontal orientation of Henle fibers facilitates cyst formation. Retinal traction generally leads to retinal deformation and consequently to development of macular edema (cystoids, focal, diffused or mixed). Metabolic, inflammatory and vascular factors play an important role in pathogenesis of ME. Cystoid cavities are zones filled with fluid surrounded by Müller's cells. Fluid can accumulate in all of the retinal layers, but most frequently between outer plexiform layer and external limiting membrane $[8,21]$. OCT shows macular edema as retinal layering followed by presence of intraretinal cavities and absence of backreflection.

One of the advantages of OCT is its ability to provide precise quantitative data concerning macular thickness, during assessment and follow up stages. Repeated OCT is an irreplaceable method for monitoring treatment effects. OCT provides means of measuring thickness and localization as well as size of intraretinal cysts during the evolution of ME.

Figure 5a shows elevated foveal contours due to presence of large, fluid filled, intraretinal cysts measuring $713 \mu \mathrm{m} \times 432 \mu \mathrm{m}$ surrounded by two smaller cysts one of which is separated by Müller cells. Retinal thickness map (Figure 5b) shows macular topography where red color with yellow border represents pathological macular thickening. Figure $5 \mathrm{c}$ shows average thickness of certain sectors expressed both numerically $(\mu \mathrm{m})$ and color-coded with a peack of macular thickening located centrally and temporally from fovea. Three follow up scans taken at same cross-section (Figure 6a) are showing evolution of cystoid macular edema. There is a noticeable empty, clearly bordered and irregular in shape intraretinal space which corresponds to a fluid filled cyst with minor subsensory effusion. Size of cyst varies; initially there is an increase, subsequently followed by decrease in volume. Structurally there are no retinal changes in the area surrounding the cyst. Inner/outer photoreceptor layer is missing at the base of the cyst. Figure $6 \mathrm{~b}$ shows changes of average macular thickness on the above mentioned examinations, while Figure $6 \mathrm{c}$ shows macular thickening at specific points/sectors upon separate examinations thus providing means for assessing deviation from normal as well as their correlation.

Lamellar hole is formed during evolution of uveitic macular complications in which ERM-foveal traction also plays an important part. ERM combined with lamellar hole is characterized by gradient of foveal contour, layering and partial decrease of retinal thickness, while outer retinal layer remains present above the RPE. Evolution of $\mathrm{ERM}$, presence of ME, changes of thickness, surface roughness and structures and layer configuration of retinal tissue can be analyzed via OCT [20]. Foveal contour showed in Figure 7 is deep, funnel-shaped and temporally steep. There is predominant retinal layering in the nasal region. Preserved outer retinal layers, $84 \mu \mathrm{m}$ in thickness, are noted at the bottom of lamellar hole. ERM is seen as a discrete highly reflective line adherent to the inner surface of the retina and discontinuous in the foveal region. Figure 8 shows deepened fovea with preserved tissue at the vary base of lamellar hole. Due to focal EMR traction, inner retinal surface is temporally serrated. OCT performed a year later shows progression of disease. Nasal parts of inner retinal layer are under severe focal ERM traction leading to retinal layering and fluid buildup in form of optically void cysts.

Localized inflammation of retina can occur at both choriocapillar and RPE levels, which can lead to degradation of outer hematoretinal barrier and subsequently to photoreceptor layer discontinuity and subretinal fluid accumulation. Foveal contour as well as retinal layers 
Citation: Paovic J, Paovic P, Paovic AS, Sredovic V (2013) Diagnostic Procedures and Follow Up of Macular Microstructural Changes in Patients with Uveitis as Seen on Optical Coherence Tomography. J Cytol Histol 5: 211. doi:10.4172/2157-7099.1000211

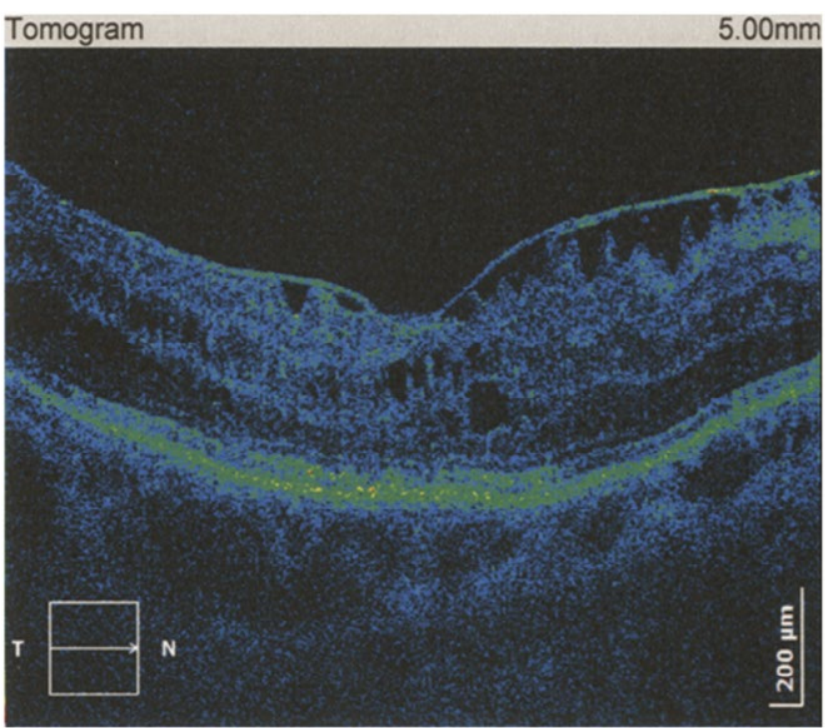

Figure 3: OCT scan, epiretinal membrane and cystoid macular edema.

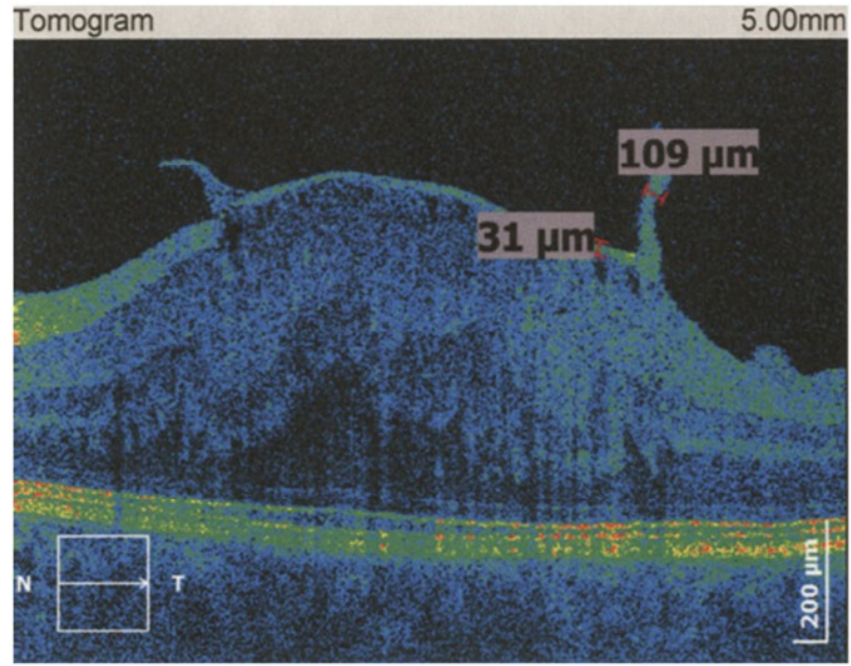

Figure 4: OCT scan, severe epiretinal membrane and diffused macular edema.

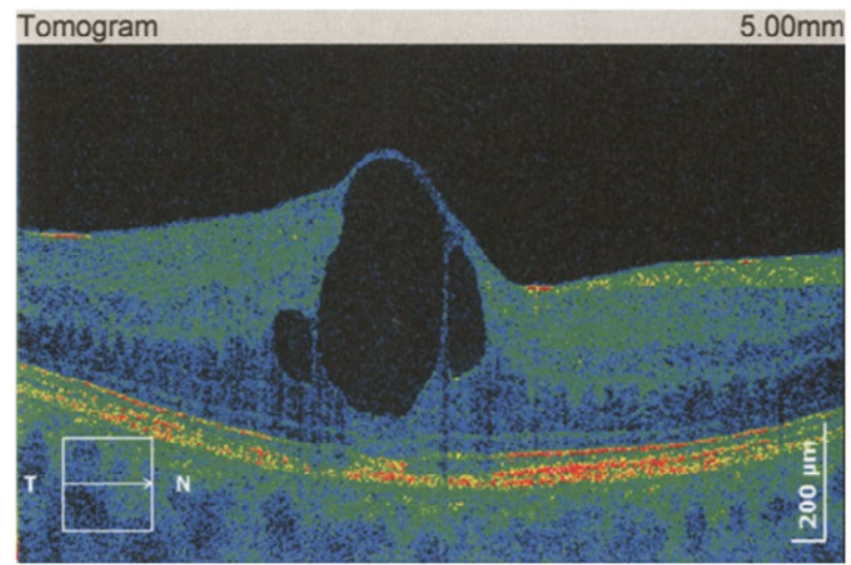

Figure 5a: OCT scan, cystoid macular edema. configuration are preserved (Figure 9a). Photoreceptors are subfoveally disrupted and reach the external limiting membrane. Three months following initial examination (Figure 9b) OCT scan reveals a barely noticeable break and a recovering photoreceptor layer. There is no noted retinal discontinuity.

OCT macular scan provides precise and quick diagnosis of delicate microstructural retinal changes in high definition which can't be obtained during the common clinical examination. Evolution, changes and treatment effect(s) followed via OCT are of great importance in clinical practice. OCT method is a noninvasive technique that can be repeated without any health risks, is suitable for children and during pregnancy while facilitating correlation and comparison of various data points. On the other hand, techniques such as FA and ICG are invasive, and don't provide enough information on macular structural changes in patients with uveitis. Biopsy and histological examination are considered as highly invasive techniques, applied only when other procedures have been exhausted and are not used as a method of follow up. Histological changes of macular tissue can be proven only in enucleated eyes (Figure 10). Immunohistochemical changes of uveoretinal tissue which are of great importance for research in uveitic pathogenesis can be tested and monitored on experimental laboratory models (Figures 11 and 12) [21-24].

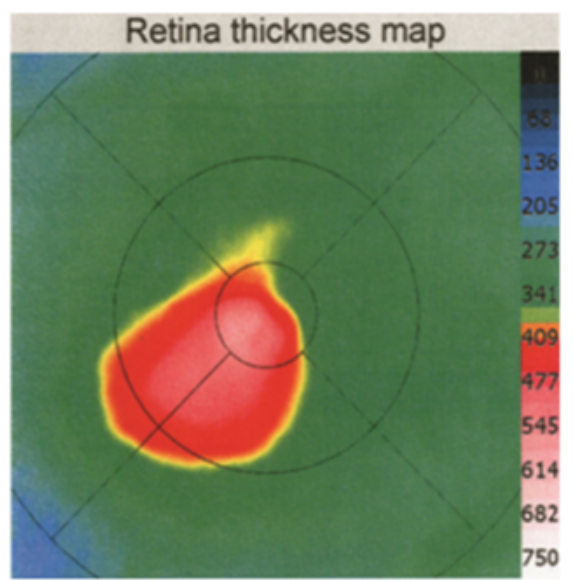

Figure 5b: Retinal thickness map, cystoid macular edema

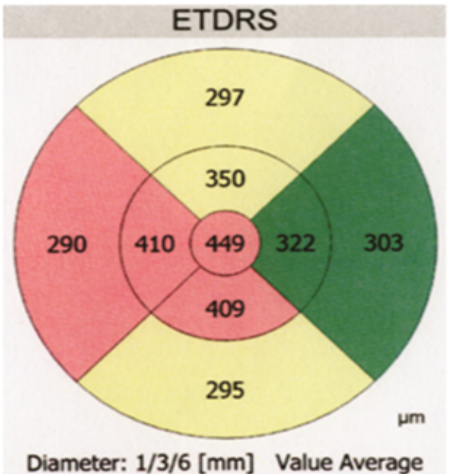

Figure 5c: Retinal thickness map - average value (diameter: 1/3/6 $\mathrm{mm})$, cystoid macular edema. 
Citation: Paovic J, Paovic P, Paovic AS, Sredovic V (2013) Diagnostic Procedures and Follow Up of Macular Microstructural Changes in Patients with Uveitis as Seen on Optical Coherence Tomography. J Cytol Histol 5: 211. doi:10.4172/2157-7099.1000211
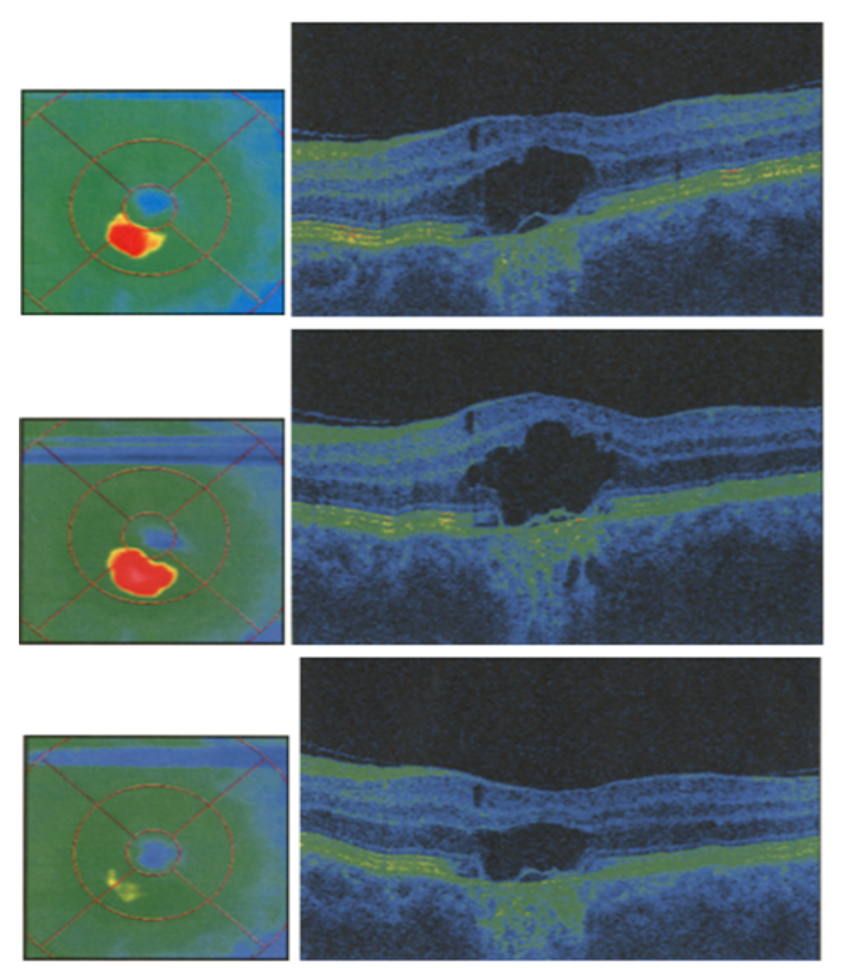

Figure 6a: OCT scans and color-coded maps, macular edema, follow up.

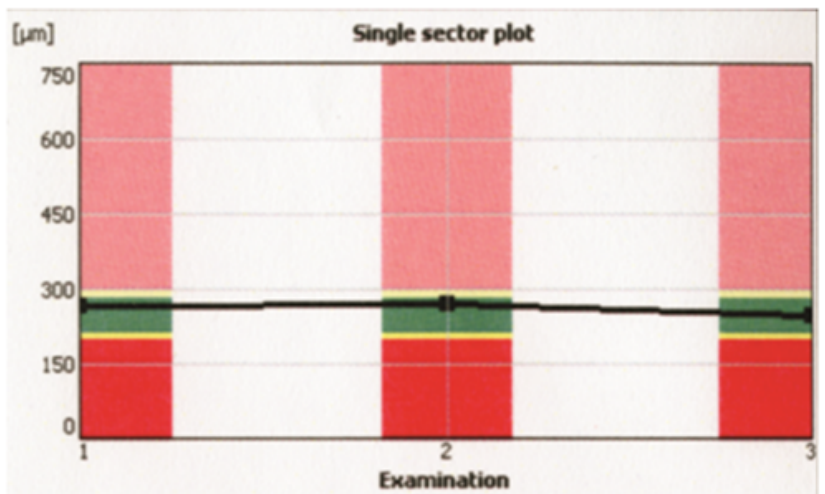

Figure 6b: Retinal thickness, single sector plot, macular edema, follow up.

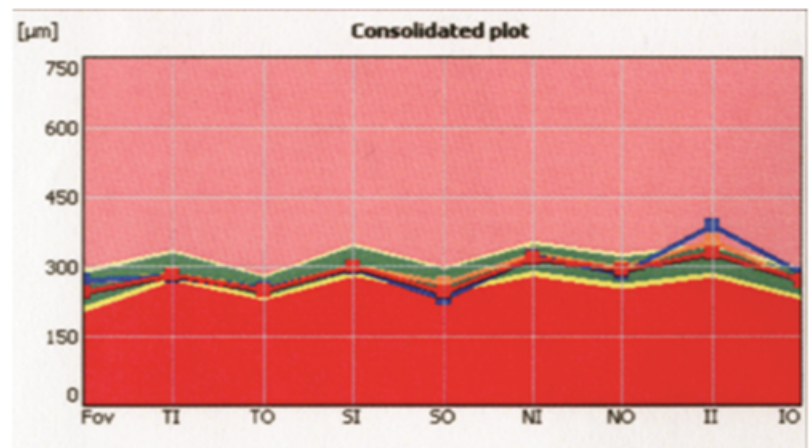

Figure 6c: Retinal thickness, consolidated plot, macular edema, follow up.

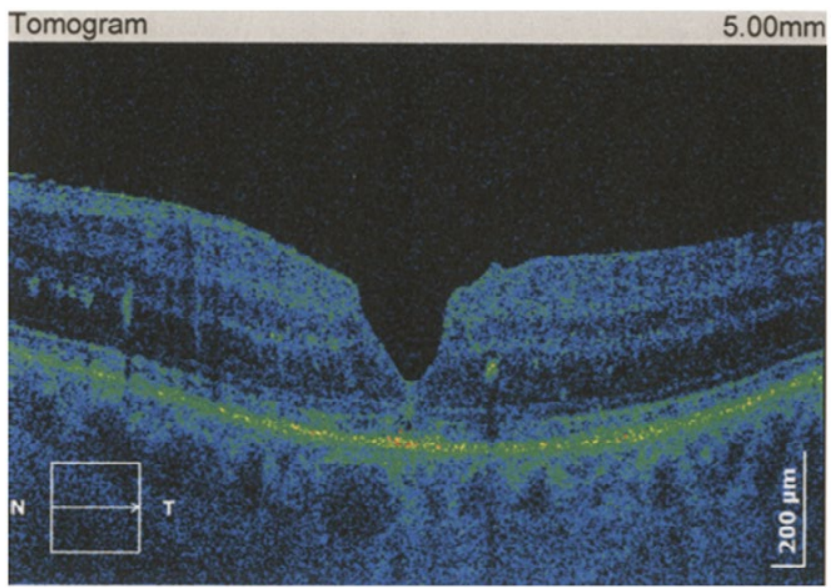

Figure 7: OCT scan, lamellar hole in patient with uveitis.

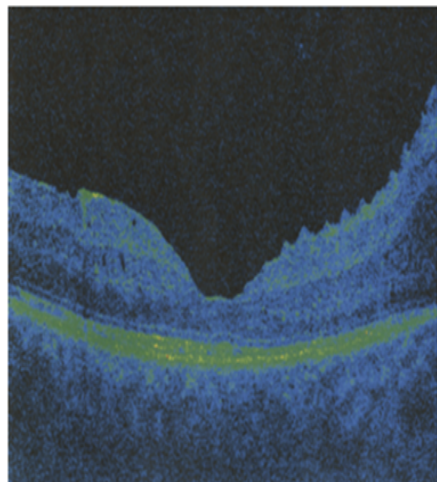

initial

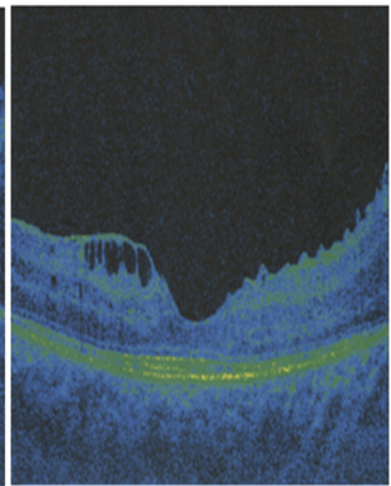

year later
Figure 8: OCT scan, lamellar hole and cystoid macular edema in patient with uveitis.

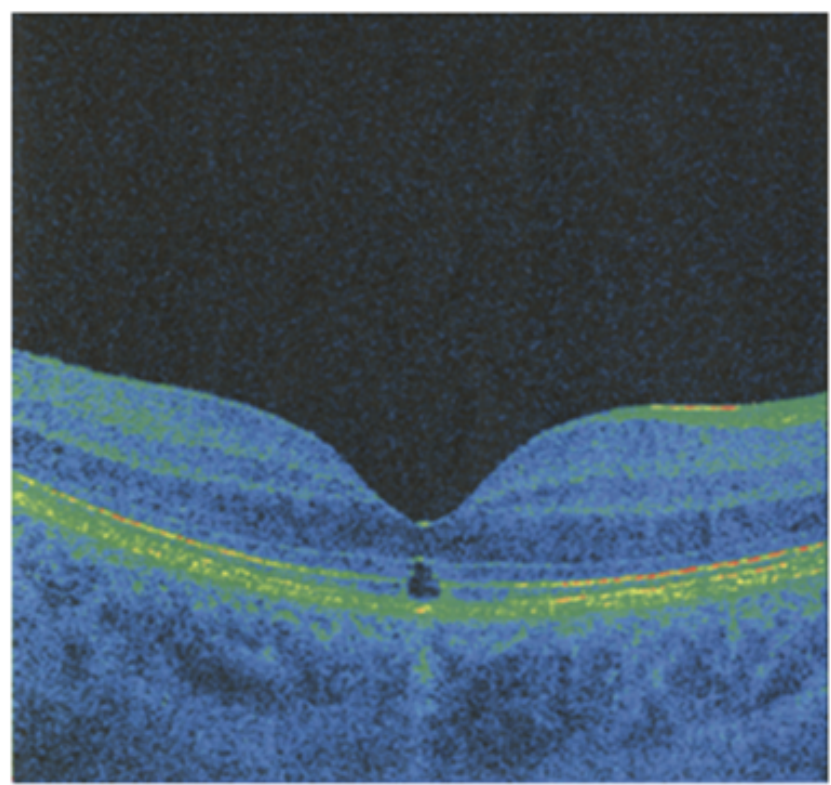

Figure 9a: OCT scan, disruption in macular photoreceptor layer. 
Citation: Paovic J, Paovic P, Paovic AS, Sredovic V (2013) Diagnostic Procedures and Follow Up of Macular Microstructural Changes in Patients with Uveitis as Seen on Optical Coherence Tomography. J Cytol Histol 5: 211. doi:10.4172/2157-7099.1000211

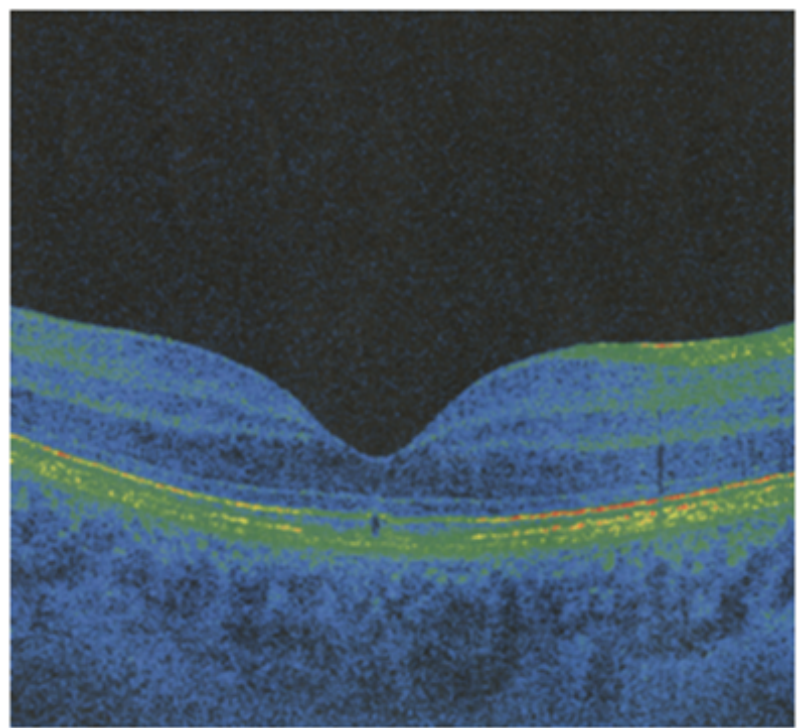

Figure 9b: OCT scan, discreet photoreceptors break at follow up.

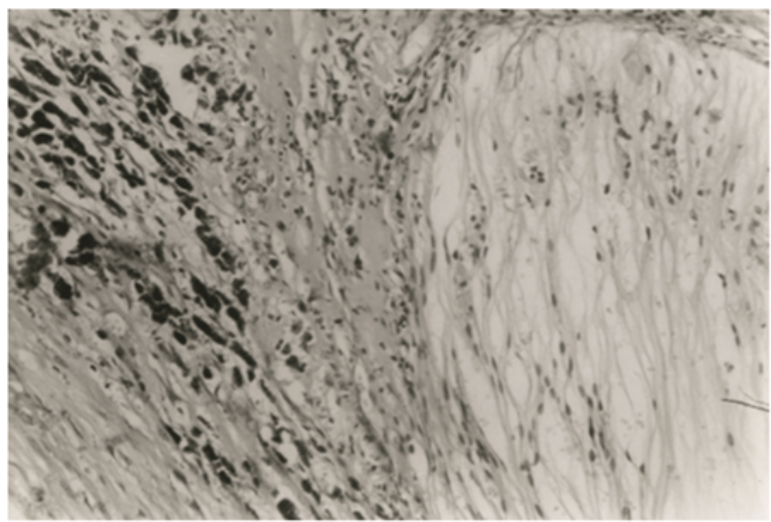

Figure 10: Microcellular infiltration in uveoretinal tissue in patient with uveitis (Behcet's disease), enucleated eye.

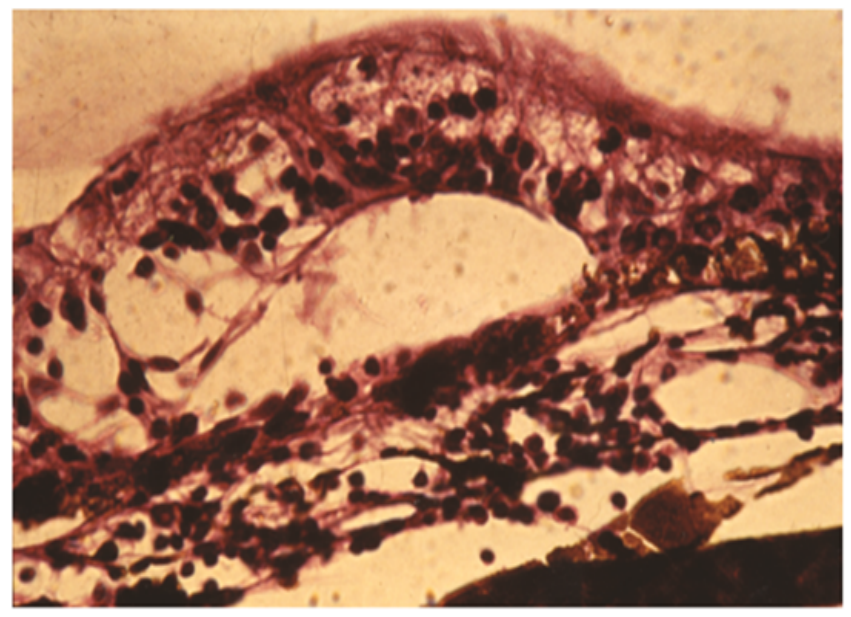

Figure 11: Immunohistochemical changes in uveoretinal tissue in experimental autoimmune uveitis caused by retinal S-antigen.

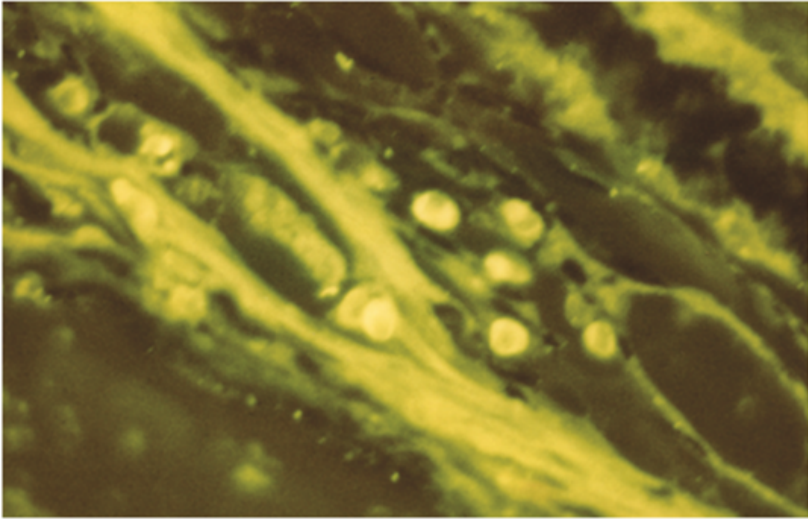

Figure 12: Pathohistological findings of plasma cells in choroidea in experimental autoimmune uveitis caused by retinal S-antigen.

\section{References}

1. Sugar EA, Jabs DA, Altaweel MM, Lightman S, Acharya N, et al. (2011) Identifying a clinically meaningful threshold for change in uveitic macular edema evaluated by optical coherence tomography. Am J Ophthalmol 152 1044-1052.

2. Hassenstein A, Bialasiewicz AA, Richard G (2000) Optical coherence tomography in uveitis patients. Am J Ophthalmol 130: 669-670.

3. Skarmoutsos F, Sandhu SS, Voros GM, Shafiq A (2006) The use of optical coherence tomography in the management of cystoid macular edema in pediatric uveitis. J AAPOS 10: 173-174.

4. Gupta V, Gupta P, Singh R, Dogra MR, Gupta A (2008) Spectral-domain Cirrus high-definition optical coherence tomography is better than time domain Stratus opticalcoherence tomography for evaluation of macular pathologic features in uveitis. Am J Ophthalmol 145: 1018-1022.

5. Kiss CG, Barisani-Asenbauer T, Maca S, Richter-Mueksch S, Radner W (2006) Reading performance of patients with uveitis-associated cystoid macular edema. Am J Ophthalmol 142: 620-624.

6. Ossewaarde-van Norel J, Camfferman LP, Rothova A (2012) Discrepancies between fluorescein angiography and optical coherence tomography in macular edema in uveitis. Am J Ophthalmol 154: 233-239.

7. Tranos PG, Wickremasinghe SS, Stangos NT, Topouzis F, Tsinopoulos I, et al (2004) Macular edema. Surv Ophthalmol 49: 470-490.

8. Catier A, Tadayoni R, Paques M, Erginay A, Haouchine B, et al. (2005) Characterization of macular edema from various etiologies by optical coherence tomography. Am J Ophthalmol 140: 200-206.

9. Fujimoto JG, Pitris C, Boppart SA, Brezinski ME (2000) Optical coherence tomography: an emerging technology for biomedical imaging and optical biopsy. Neoplasia 2: 9-25.

10. Fujimoto JG, Brezinski ME, Tearney GJ, Boppart SA, Bouma B, et al. (1995) Optical biopsy and imaging using optical coherence tomography. Nat Med 1 : 970-972.

11. Puliafito CA, Hee MR, Lin CP, Reichel E, Schuman JS, et al. (1995) Imaging of macular diseases with optical coherence tomography. Ophthalmology 102 217-229.

12. Hee MR, Izatt JA, Swanson EA, Huang D, Schuman JS, et al. (1995) Optical coherence tomography of the human retina. Arch Ophthalmol 113: 325-332.

13. Multicenter Uveitis Steroid Treatment Trial Research Group, Kmepen JH, Altaweel MM, Holbrook JT, Jabs DA, et al. (2010) The multicenter uveitis steroid treatment trial: retionale, design and baseline characteristics. Am J Ophthalmol 149: 550-561.

14. $\mathrm{Ng} \mathrm{CH}$, Cheung N, Wang JJ, Islam AF, Kawasaki R, et al. (2011) Prevalence and risk factors for epiretinal membranes in a multi-ethnic United States population. Ophthalmology 118: 694-699. 
Citation: Paovic J, Paovic P, Paovic AS, Sredovic V (2013) Diagnostic Procedures and Follow Up of Macular Microstructural Changes in Patients with Uveitis as Seen on Optical Coherence Tomography. J Cytol Histol 5: 211. doi:10.4172/2157-7099.1000211

15. Gomes NL, Corcostegui I, Fine HF, Chang S (2009) Subfoveal pigment changes in patients with longstanding epiretinal membranes. Am J Ophthalmol 147: 865-868.

16. Oster SF, Mojana F, Brar M, Yuson RM, Cheng L, et al. (2010) Disruption of the photoreceptor inner segment/outher segment layer on spectral domain optical coherence tomography is a predictor of poor visual acuity in patients with epiretinal membranes. Retina 30: 713-718.

17. Falkner-Radler Cl, Glittenberg C, Hagen S, Benesch T, Binder S (2010) Spectral-domain optical coherence tomography for monitoring epiretinal membrane surgery. Ophthalmology 117: 798-805.

18. Nigam N, Bartsch DU, Cheng L, Brar M, Yuson RM, et al. (2010) Spectral domain optical coherence tomography for imaging ERM, retinal edema, and vitreomacular interface. Retina 30: 246-253.
19. Nazari H, Dustin L, Heussen FM, Sadda S, Rao NA (2012) Morphometric spectral-domain optical coherence tomography features of epiretinal membrane correlate with visual acuity in patients with uveitis. Am J Ophthalmol 154: 78-86.

20. Antcliff RJ, Marshall J (1999) The pathogenesis of edema in diabetic maculopathy. Semin Ophthalmol 14: 223-232.

21. Piccolino FC, Borgia L (1994) Central serous chorioretinopathy and indocyanine green angiography. Retina 14: 231-242.

22. Hee MR, Puliafito CA, Wong C, Reichel E, Duker JS, et al. (1995) Optical coherence tomography of central serous chorioretinopathy. Am J Ophthalmo 120: 65-74.

23. Wang M, Sander B, Lund-Andersen H, Larsen M (1999) Detection of shallow detachments in central serous chorioretinopathy. Acta Ophthalmol Scand 77: 402-405.

24. Stanojevic-Paovic A. Uveitisi. Medicinski fakultet Beograd, 2008, poglavlje Specijalni deo, str. 75 